\title{
Simulations of a Liquid Hydrogen Inducer at Low-Flow Off-Design Flow Conditions
}

\author{
A. Hosangadi, ${ }^{*}$ and V. Ahuja, ${ }^{\dagger}$ And R.J. Ungewitter. ${ }^{\ddagger}$ \\ Combustion Research and Flow Technology, Inc. (CRAFT Tech), Pipersville, PA 18947
}

The ability to accurately model details of inlet back flow for inducers operating at lowflow, off-design conditions is evaluated. A sub-scale version of a three-bladed liquid hydrogen inducer tested in water with detailed velocity and pressure measurements is used as a numerical test bed. Under low-flow, off-design conditions the length of the separation zone as well as the swirl velocity magnitude was under predicted with a standard $k$ - $\varepsilon$ model. When the turbulent viscosity coefficient was reduced good comparison was obtained at all the flow conditions examined with both the magnitude and shape of the profile matching well with the experimental data taken half a diameter upstream of the leading edge. The velocity profiles and incidence angles at the leading edge itself were less sensitive to the back flow length predictions indicating that single-phase performance predictions may be well predicted even if the details of flow separation modeled are incorrect. However, for cavitating flow situations the prediction of the correct swirl in the back flow and the pressure depression in the core becomes critical since it leads to vapor formation. The simulations have been performed using the CRUNCH CFD ${ }^{\circledR}$ code that has a generalized multi-element unstructured framework and an advanced multi-phase formulation for cryogenic fluids. The framework has been validated rigorously for predictions of temperature and pressure depression in cryogenic fluid cavities and has also been shown to predict the cavitation breakdown point for inducers at design conditions.

\section{Nomenclature}

$c_{m}, c_{g}, c_{L} \quad$ Speed of sound in mixture, gas, liquid

$D_{\mathrm{v}} \quad$ Viscous flux vector

$E, F, G \quad$ Flux vectors

$h_{m} \quad$ Mixture enthalpy

$k, \varepsilon \quad$ Turbulent kinetic energy, turbulent dissipation rate

$K_{f}, K_{b}$ Rate constants for vapor and liquid formation

$m_{t} \quad$ Rate of vapor mass formation

$p \quad$ Pressure

$\rho_{m}, \rho_{g}, \rho_{L} \quad$ Density of mixture, gas, liquid

$Q, E, S \quad$ Vector of conservative variables

$Q_{v} \quad$ Vector of primitive variables

$S \quad$ Source terms

$S_{g} \quad$ Cavitation source term

$T_{\text {sat }} \quad$ Temperature saturation

$u, v, w \quad \mathrm{x}, \mathrm{y}, \mathrm{z}-$ components of velocity

$\mathrm{D} H_{v} \quad$ Latent heat formation of vapor

$\phi_{g} \phi_{L} \quad$ Void fraction of gas, liquid

$\sigma_{k}, \sigma_{\epsilon}, \mathrm{C}_{1}$ and $\mathrm{C}_{2}$ Modeling constants

\footnotetext{
* Principal Research Scientist, 6210 Keller's Church Road, Pipersville, PA 18947, AIAA Member.

† Research Scientist, 6210 Keller's Church Road, Pipersville, PA 18947, Senior AIAA Member.

‡ Research Scientist, 6210 Keller's Church Road, Pipersville, PA 18947, AIAA Member.
} 


\section{Introduction}

$\mathrm{O}$ UR interest is in the development of a computational framework to simulate cavitating liquid rocket turbomachinery that employ cryogenic working fluids. Liquid rocket systems are a subset of a broader class of pumps (e.g. refrigerant systems, boiler feed pumps, etc) where the operating temperature is elevated relative to the critical temperature of the fluid and thermodynamic effects of cavitation play an important role. At these operating temperatures, the ratio of liquid to vapor density is lower and consequently more liquid mass has to vaporize to sustain a cavity. Therefore evaporative cooling effects are more pronounced and result in the lowering of the mean fluid temperature in the cavitating region. Since the fluid thermodynamics properties (i.e. vapor pressure, density) are a strong function of temperature at these conditions, thermal effects suppress cavitation and lower the cavity pressure in a mean sense. Typically this results in improved mean performance of cryogenic pumps; liquid hydrogen systems being an extreme example where the pump may continue to generate head even when the fluid is boiling at the inlet.

The thermal effects of cavitation were studied extensively by numerous researchers through the 1970's including: Hord ', Stahl and Stepanoff ${ }^{2}$, Ruggeri and Moore ${ }^{3}$, Holl ${ }^{4}$, and Brennen ${ }^{5}$ among others. Stahl and Stepanoff ${ }^{2}$ were the first to estimate head depression $\left(\Delta H_{v}\right)$ values due to thermodynamic effects using the socalled 'B-factor' method based on a quasi-static theory where the temperature depression was estimated in terms of the ratio of the vapor volume to liquid volume. They provided a graph to evaluate NPSH corrections for hydrocarbons based on this methodology. More elaborate correlations, which included dynamic effects were given later by Hord ${ }^{1}$, Ruggeri and Moore ${ }^{3}$, and Holl ${ }^{4}$. They collected extensive experimental data of cavity pressure and temperature depressions for a variety of model shapes and fluids and correlated the results using variants of the Bfactor theory. The semi-empirical procedures outlined by Ruggeri and Moore ${ }^{3}$ continue to be used as an engineering tool for predicting the thermodynamic depression in pumps. A more rigorous numerical procedure was developed by Cooper ${ }^{6}$ where a baratropic equation of state was used to define the two-phase mixture and thermal effects were evaluated with a resulting non-dimensional vaporization parameter. Most of these techniques, however, require some degree of empiricism. Therefore, from a more fundamental modeling perspective, this discussion highlights the need for a generalized formulation that takes the energy balance into account when simulating cavitation for cryogenic flows.

For turbomachinery simulations, the majority of cavitating pump simulations presented in the literature are limited to simulating idealized liquids (no thermodynamic effects) at design conditions where large-scale inlet backflow is not present. Typical simulations show comparisons with data at design conditions for the head coefficient and the critical $\mathrm{N}_{\mathrm{ss}}$ number at which performance breakdown occurs (Hosangadi et al. ${ }^{7}$, Athavale and Singhal ${ }^{8}$, Dupont and Okamura ${ }^{9}$, Medvitz ${ }^{10}$ ). However flows at off-design conditions, where large scale unsteadiness and high dynamic pressure loads are observed, cannot at this point be reliably predicted Simulation of cavitation instabilities and rotational cavitation modes in pumps have not been simulated by any group to the best of our knowledge.

The development of a framework for cavitating, cryogenic fluids with real fluid property variations was addressed in our earlier work (Hosangadi and Ahuja ${ }^{11}$ ) and is an extension of our model for idealized fluids (Ahuja et al. ${ }^{12}$ ). To simulate this class of flows, a generalized multi-phase formulation has been developed that rigorously models thermal effects of phase change and the accompanying property variations. Thermal equilibrium is assumed and fluid thermodynamic propertics are specified along the saturation line using the NIST-12 databank. The multiphase model has been implemented in the CRUNCH CFD ${ }^{\circledR}$ code, which is a multi-element based unstructured code ${ }^{13,14}$. The underlying philosophy in the CRUNCH CFD ${ }^{\circledR}$ code is to tailor the grid topology to resolving the dominant flow phenomena and the structural complexity of the problem. This is achieved by utilizing a combination of hexahedral, tetrahedral, prismatic and pyramidal elements in mesh construction. Such a framework is particularly attractive for complex turbomachine configurations, since high quality grids can be generated very efficiently with minimum skewness.

The multi-phase, real-fluid formulation was rigorously validated for cavitation in liquid nitrogen and hydrogen (Hosangadi and Ahuja ${ }^{11}$ ). Predictions of temperature and pressure depression for flow over hydrofoils were compared with detailed experimental data obtained by Hord 1. The framework was subsequently applied to the study of the full-scale LOX inducer (Hosangadi et al. ${ }^{15}$ ) whose cavitating performance at design conditions was compared to that of sub-scale water test. The increase in suction performance of the LOX pump resulting from thermal effects was identified at design flow conditions where large scale flow separation is not present and the flow is relatively steady.

In the present effort, our interest is in the simulation of flowfields at off-design, low-flow conditions where large scale separation is present and a swirling back flow extends upstream of the leading edge. The backflow 
results in the acceleration of the meridional velocity in the core and the generation of a radial pressure gradient with a low pressure core to sustain the swirl. Under cavitating conditions the separated flow can interact with the phase change mechanism to generate cavitation surge instability wherein large-amplitude axial fluctuations of pressure and mass flow thru the pump may be observed. While the simulation of cavitating flow at these off-design conditions is our ultimate goal, the first step is to validate the ability to predict the single-phase back flow.

Here we focus on evaluating the ability of a Navier-Stokes methodology with a two-equation $k-\varepsilon$ model to accurately predict the details of the single-phase flowfield upstream of the leading edge. The inducer chosen for our study was a three-bladed liquid hydrogen inducer designed at NASA Marshall (Thornton ${ }^{16}$ ) using the Agile Engineering Design System tool of ConceptsNREC, and subsequently tested at ConceptsNREC in water for a subscale configuration (Japikse and Baun, ${ }^{17}$ ). Detailed measurements of velocity and pressure are available upstream of the leading edge while the flow deviation angle downstream of the trailing edge was also measured. As we shall discuss later when backflow was present, the standard $k-\varepsilon$ turbulence model overpredicted the turbulence viscosity in the separated region and hence underpredicted the back flow region. Good comparison was obtained when the turbulence constants were altered to reduce the level of turbulent viscosity indicating the sensitivity of the separation zone to this quantity. We are currently involved in evaluating if other turbulence models such as the realizable $k-\varepsilon$ model perform better under these flow conditions.

\section{Multi-Phase Equation System}

The multiphase equation system is written in vector form as:

$$
\frac{\partial Q}{\partial t}+\frac{\partial E}{\partial x}+\frac{\partial F}{\partial y}+\frac{\partial G}{\partial z}=S+D_{v}
$$

The multiphase equation system is written in vector form as:

Here $Q$ is the vector of dependent variables, $E, F$ and $G$ are the flux vectors, $S$ the source terms and $D_{v}$ represents the viscous fluxes. The viscous fluxes are given by the standard full compressible form of Navier Stokes equations. The vectors $\mathrm{Q}, \mathrm{E}$ and $\mathrm{S}$ are given below with a detailed discussion on the details of the cavitation source terms to follow later:

$$
Q=\left(\begin{array}{c}
\rho_{m} \\
\rho_{m} u \\
\rho_{m} v \\
\rho_{m} w \\
\rho_{g} \phi_{g} \\
\rho_{m} k \\
\rho_{m} \varepsilon
\end{array}\right) \quad E=\left(\begin{array}{c}
\rho_{m} u \\
\rho_{m} u^{2}+P \\
\rho_{m} u v \\
\rho_{m} u w \\
\rho_{g} \phi_{g} u \\
\rho_{m} k u \\
\rho_{m} \varepsilon u
\end{array}\right) \quad S=\left(\begin{array}{c}
0 \\
0 \\
0 \\
0 \\
S_{g} \\
S_{k} \\
S_{\varepsilon}
\end{array}\right)
$$

Here, $\rho_{\mathrm{m}}$ and $h_{m}$ are the mixture density and enthalpy respectively, and $\phi_{\mathrm{g}}$ is the volume fraction or porosity of the vapor phase. The mixture energy equation has been formulated with the assumption that the contribution of the pressure work on the mixture energy is negligible which is a reasonable assumption for this flow regime. The source term for the vapor phase arises from rate of vapor mass generation due to cavitation $m_{t}$ and the corresponding source term for the energy equation is given as $m_{i} \cdot h_{f g}$ where $h_{f g}$ is the change in enthalpy resulting from the phase change and is a function of the local fluid temperature.

The mixture density and gas porosity are related by the following relations locally in a given cell volume:

$$
\begin{gathered}
r_{m}=r_{g} f_{g}+r_{L} f_{L} \\
1=\phi_{g}+\phi_{L}
\end{gathered}
$$


where $\rho_{\mathrm{g}}, \rho_{\mathrm{L}}$ are the physical material densities of the gas and liquid phase respectively and in general are functions of both the local temperature and pressure.

Thus far we have not made any statements defining the temperatures characterizing the liquid and vapor. In general, the liquid and vapor may not be in equilibrium locally and can have independent temperatures. Examination of temperature and pressure data for cavitation in Freon by Ruggeri 18 reveals that the saturation vapor pressure corresponding to the local fluid temperature in fact matches the local pressure measurement. This indicates local thermodynamic equilibrium that is exploited to significantly simplify Eqn. (1). The thermodynamic properties of the liquid and vapor in the cavity may now be defined by a single variable; the saturation temperature $T_{\text {sat }}$. Hence, all thermodynamic properties (density, vapor pressure, viscosity, etc) of both the liquid and the vapor phase may be generated as a tabular function of the saturation temperature. In our study here, these properties were generated from the Standard thermodynamic database 12 available from NIST for pure fluids.

The equation system as formulated in Eqn. (1) is very stiff since the variations in density are much smaller than the corresponding changes in pressure. Therefore to devise an efficient numerical procedure we wish to transform Eqn. (1) to a pressure based form where pressure rather than density is the variable solved for. An acoustically accurate two-phase form of Eqn. (1) is first derived, followed by a second step of time-scaling or preconditioning to obtain a well-conditioned system. We begin by defining the acoustic form of density differential for the individual gas and liquid phase as follows:

$$
d \rho_{g}=\frac{1}{c_{g}^{2}} d P, d \rho_{l}=\frac{1}{c_{L}^{2}} d P
$$

Here $c_{g}$ is the isothermal speed of sound $\left(\frac{\partial P}{\partial \rho_{g}}\right)_{T}$ in the pure gas phase, and $c_{\mathrm{L}}$ is the corresponding isothermal speed of sound in the liquid phase, which is a finite-value. We note that in Eqn. (5) the variation of the density with temperature has been neglected in the differential form. This assumption was motivated by the fact that the temperature changes are primarily due to the source term and not by the pressure work on the fluid i.e. the energy equation is a scalar equation. This simplifies the matrix algebra for the upwind flux formulation significantly, at the potential expense of numerical stability in a time-marching procedure. However, more importantly, there is no impact on the accuracy since the fluid properties themselves are taken directly from the thermodynamic data bank for each fluid.

Following the discussion above, the differential form of the mixture density $\rho_{m}$ using Eqn. (5) is written as,

$$
\begin{aligned}
& d \rho_{m}=\left(\rho_{g}-\rho_{L}\right) d \phi_{g}+\frac{1}{c_{\phi}^{2}} d P \\
& \left(\frac{1}{c_{\phi}^{2}}=\frac{\phi_{g}}{c_{g}{ }^{2}}+\frac{\phi_{L}}{c_{L}{ }^{2}}\right)
\end{aligned}
$$

Here, $c_{\phi}$ is a variable defined for convenience and is not the acoustic speed, $c_{m}$, in the mixture, which will be defined later. Using Eqn. (6), Eqn. (1) may be rewritten as:

$$
\Gamma \frac{\partial Q_{v}}{\partial t}+\frac{\partial E}{\partial x}+\frac{\partial F}{\partial y}+\frac{\partial G}{\partial z}=S+D_{v}
$$

and,

$$
Q_{v}=\left[p, u, v, w, \phi_{g}, k, \varepsilon\right]^{T}
$$


The numerical characteristics of the Eqn. (7) are studied by obtaining the eigenvalues of the matrix, $\left[\Gamma^{-1}\left(\frac{\partial \mathrm{E}}{\partial Q_{v}}\right)\right]$. The eigenvalues of the system are derived to be:

$$
\Lambda=\left(u+c_{m}, u-c_{m}, u, u, u, u, u\right)
$$

where $c_{m}$ turns out to be the well-known, harmonic expression for the speed of sound in a two-phase mixture and is given as:

$$
\frac{1}{c_{m}{ }^{2}}=\rho_{m}\left[\frac{\phi_{g}}{\rho_{g} c_{g}{ }^{2}}+\frac{\phi_{L}}{\rho_{L} c_{L}{ }^{2}}\right]
$$

The behavior of the two-phase speed of sound indicates that at either limit the pure single-phase acoustic speed is recovered. However, away from the single-phase limits, the acoustic speed rapidly drops below either limit value and remains at the low-level in most of the mixture regime. As a consequence, the local Mach number in the interface region can be large even in low speed flows. To obtain an efficient time-marching numerical scheme, preconditioning is now applied to the system in Eqn. (7), in order to rescale the eigenvalues of the system so that the acoustic speeds are of the same order of magnitude as the local convective velocities.

The effects of turbulent mixing are accounted for by employing a two-equation $k$ - $\varepsilon$ model. The turbulent viscosity is obtained by solving transport equations for the turbulent kinetic energy and its dissipation rate as follows,

$$
\begin{gathered}
\frac{\partial \rho k}{\partial t}+\frac{\partial}{\partial x_{i}}\left(\rho u_{i} k-\left(\mu+\frac{\mu_{T}}{\sigma_{k}}\right) \frac{\partial k}{\partial x_{i}}\right)=P_{k}-\rho \varepsilon+S_{k} \\
\frac{\partial \rho \varepsilon}{\partial t}+\frac{\partial}{\partial x_{i}}\left(\rho u_{i} \varepsilon-\left(\mu+\frac{\mu_{T}}{\sigma_{\mathcal{E}}}\right) \frac{\partial \varepsilon}{\partial x_{i}}\right)=C_{1} f_{1} P_{k}-C_{2} f_{2} \rho \mathcal{E}+S_{\mathcal{E}} \\
P_{k}=\tau_{i j} \frac{\partial u_{i}}{\partial x_{j}}, \tau_{i j}=-\frac{2}{3} \rho k+2 \mu_{T} *\left(S_{i j}-\frac{1}{3} \frac{\partial u_{k}}{\partial x_{k}} \delta_{i j}\right), \\
\mu_{T}=C_{\mu} f_{\mu} \rho \frac{k^{2}}{\varepsilon}
\end{gathered}
$$

where, $\sigma_{k}, \sigma_{\varepsilon} \mathrm{C}_{1}$ and $\mathrm{C}_{2}$ are modeling constants given as follows:

$$
\begin{aligned}
& c_{\mu}=0.09, \sigma_{k}=1.4, \sigma_{\varepsilon}=1.4, \\
& c_{1}=1.44, c_{2}=1.92, c_{3}=2.9556
\end{aligned}
$$

For the inducer calculations presented here a wall-function procedure was used to specify the boundary conditions for the turbulence kinetic energy and the dissipation rate at the blade surfaces.

\section{A. Cavitation Source Terms}

In the present effort, the cavitation source term is defined via a simplified non-equilibrium, finite rate form as follows:

$$
m_{t}=K_{f} \rho_{L} \phi_{L}+K_{b} \rho_{g} \phi_{g}
$$

where the constant $\mathrm{K}_{\mathrm{f}}$ is the rate constant for vapor being generated from liquid in a region where the local pressure is less than the vapor pressure. Conversely, $K_{b}$ is the rate constant for reconversion of vapor back to liquid in 
regions where the pressure exceeds the vapor pressure. Here, the rate constants are specified using the form given by Merkle 19.

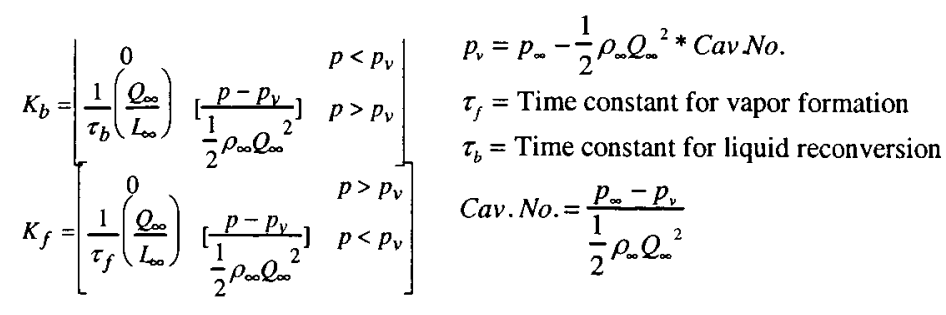

We note that for steady attached cavitation this simplified form may be adequate since the cavitation time scales do not interact with the fluid time scales if the cavitation rate constants are fast enough. For unsteady cavitation modeling, however, it becomes essential to integrate bubble dynamics within a dense cloud framework wherein both the number density and mean local radius of the bubbles in an evolving cloud are tracked. The development of a more rigorous non-equilibrium source term model is a topic of ongoing research.

\section{Validation Study for Cavitation in Cryogenic Fluids}

The multi-phase formulation described above has been validated extensively by simulating experiments by Hord ${ }^{1}$ on a cavitating hydrofoil. Hord performed sub-scale tests using both liquid nitrogen and hydrogen in a blowdown tunnel. The details of the tunnel and the hydrofoil geometry are given in Figure 1 . The tunnel width is 1 inch while the hydrofoil width is 0.312 inches.

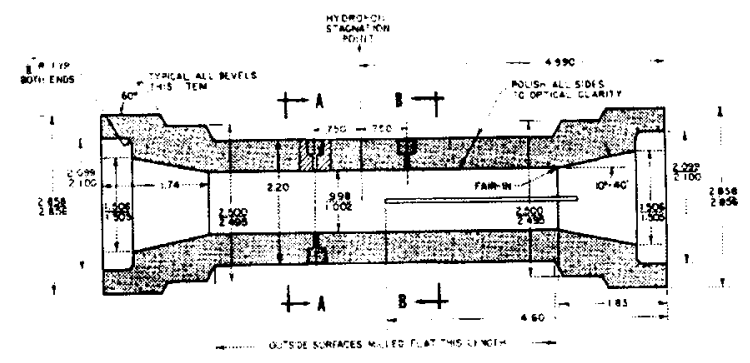

Quarter Caliber Hydrofoil with a Tapered Diffuser

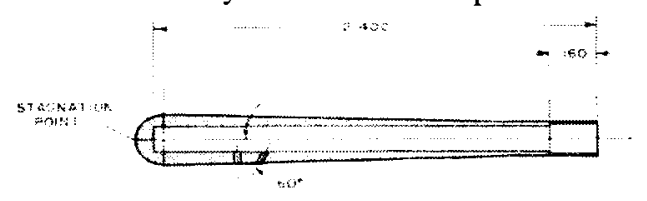

Tunncl Geometry

Figure 1. Details of Experimental Set-up for Cavitating Hydrofoil (Taken from Hord, 1).

Hence considerable blockage effects from the tunnel wall are present and this necessitated modeling the tunnel geometry in the simulations. To ensure that the tunnel blockage effects were being correctly modeled we performed single-phase, non-cavitating simulations and have compared it to Hord's non-cavitating data in Figure 2. Excellent agreement is obtained giving confidence that the tunnel interaction is being captured. We note that the single-phase solution is insensitive to the Reynolds number since both liquid hydrogen and nitrogen show identical pressure profiles.

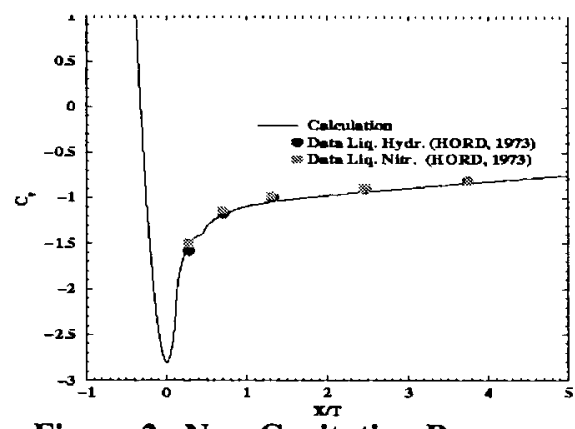

Figure 2. Non-Cavitating Pressure Distribution on Hydrofoil with Tunnel Blockage Modeled (Hord ${ }^{\mathbf{1}}$ ). 
Cavitating results in liquid nitrogen for one flow condition are discussed here. We refer the reader to Hosangadi and Ahuja ${ }^{11}$ for additional validation studies in both liquid nitrogen and hydrogen. The operating range of liquid nitrogen varies from roughly $70 \mathrm{~K}-100 \mathrm{~K}$. The variation of properties along the saturation line for liquid and vapor densities as well as the vapor pressure are shown in Figure 3. At $89 \mathrm{~K}$, the slope of vapor pressure curve indicates a $16 \mathrm{KPa}$ increase for a $1 \mathrm{~K}$ change in temperature.

The general characteristics of the cavitating flowfield are shown in Figure 4(a-d) for tunnel conditions in Run 290C (Table I). Figure 4a shows the temperature profile in the cavity. The strong temperature depression at the leading edge of the cavity is evident with the gradual temperature recovery due to condensation in the rear of the cavity. The vapor volume fraction is qualitatively compared with a typical flow visualization of the flow (note that the flow conditions at which this visualization was done are not reported in the Hord's report). The overall shape and features of the cavity appear to be similar. The computed pressure field indicates strong interaction between the cavity and the tunnel wall which is expected due to the relative scales of the geometry.

Table I. Run Conditions For Liquid Nitrogen Cases

\begin{tabular}{|c|c|c|c|c|}
\hline $\begin{array}{c}\text { Run } \\
\text { Number }\end{array}$ & $\begin{array}{c}\text { Freestream } \\
\text { Temp. (K) }\end{array}$ & $\begin{array}{c}\text { Freestream } \\
\text { Vel. (M/S) }\end{array}$ & $\begin{array}{c}\text { Cavitation } \\
\text { Number }\end{array}$ & $\begin{array}{c}\text { Cavity } \\
\text { Length (CM) }\end{array}$ \\
\hline 289C & 88.64 & 23.5 & 1.55 & 2.29 \\
\hline 290C & 83.06 & 23.9 & 1.70 & 1.9 \\
\hline $293 \mathrm{~A}$ & 77.64 & 24.0 & 1.75 & 1.52 \\
\hline 294F & 77.94 & 9.8 & 1.78 & 1.52 \\
\hline
\end{tabular}

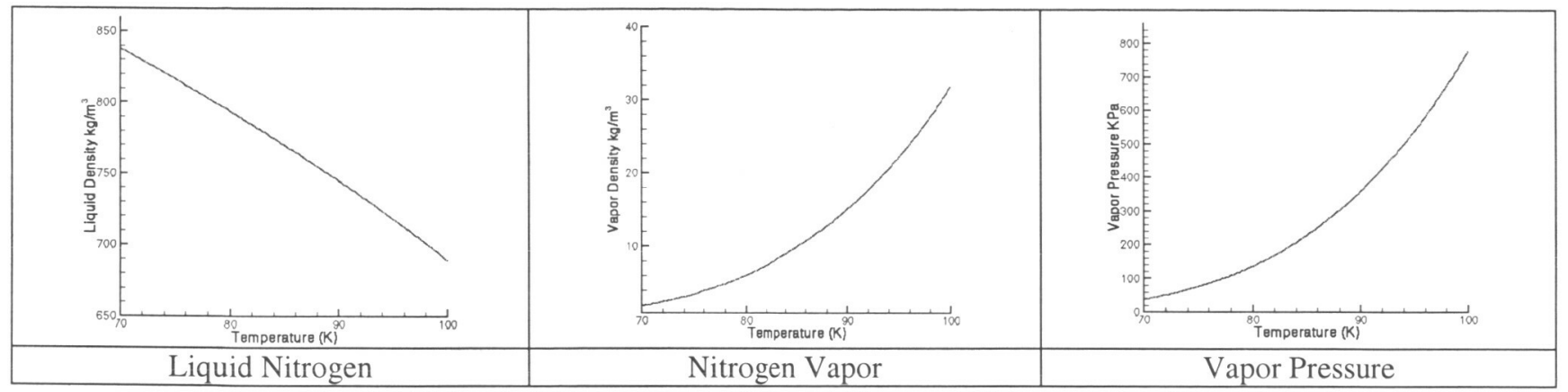

Figure 3. Physical Properties Of Liquid Nitrogen (Temperature Dependence).

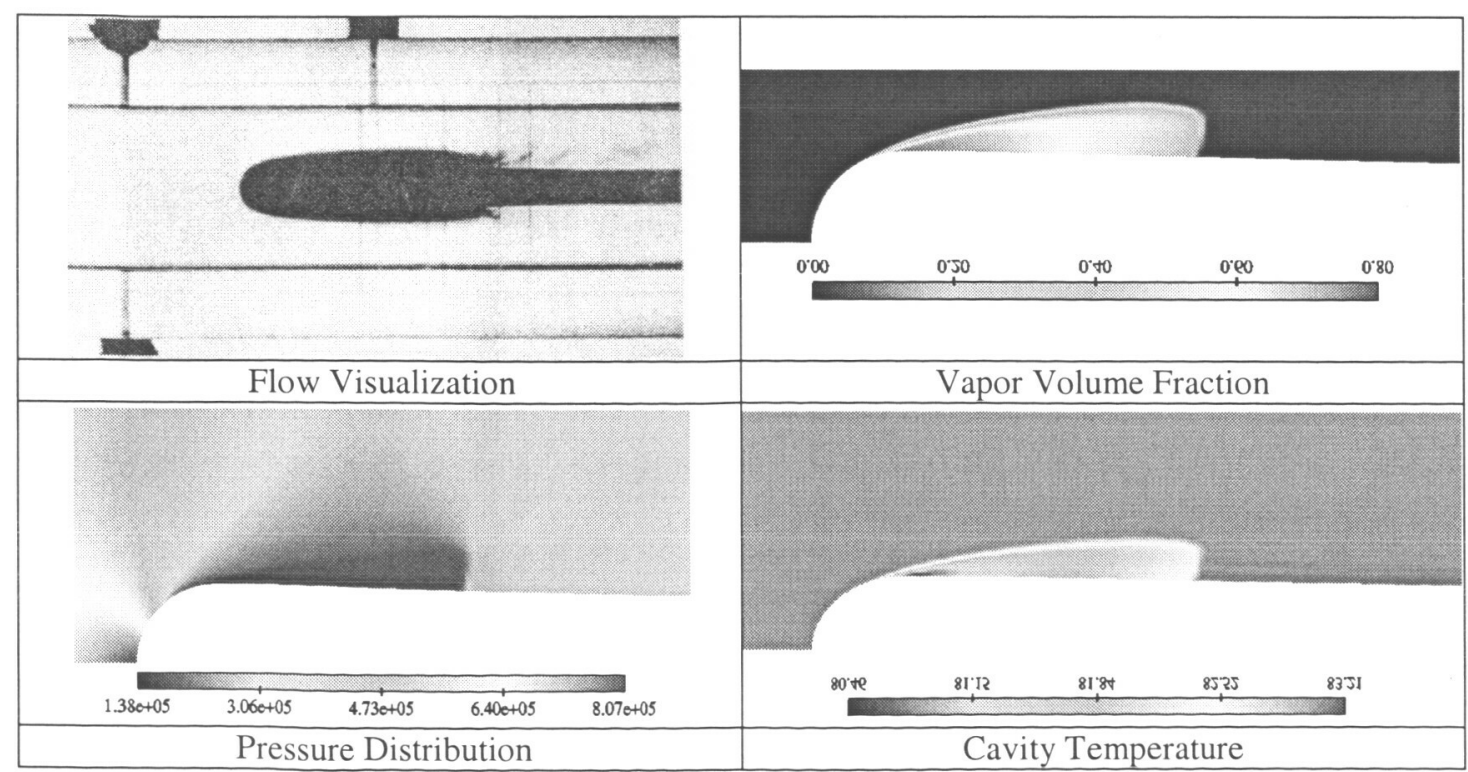

Figure 4. Cavitating Hydrofoil Flowfield For conditions of Run 290C in Liquid Nitrogen. 
The quantitative comparisons of pressure and temperature depression in the cavity are compared with experimental data in Figure 5. Note that the pressure values plotted are $\left(P-P_{\nu, \infty}\right)$. For a non-cryogenic case this value would be zero in the cavity, while values below zero in the cryogenic case indicate pressure depression due to thermal effects. In general excellent comparison is obtained for the leading edge temperature depression of approximately $2.5 \mathrm{~K}$. The temperature recovery within the cavity compares well both in their slope as well as the length of the cavity. The temperature rise in the cavity closure region shows some differences; the computed solution recovers to the freestream value more quickly than does the data, which doesn't quite fully recover to the freestream value. As per the discussion by Hord ${ }^{1}$, this probably was due to the unsteady effects in the cavity closure region whereby the thermocouples were not always enclosed in vapor giving erroneous readings. The instrumentation error given for the chromel-gold thermocouples is $0.20 \mathrm{~K}$ and the computed results are within the uncertainty of the experiments.

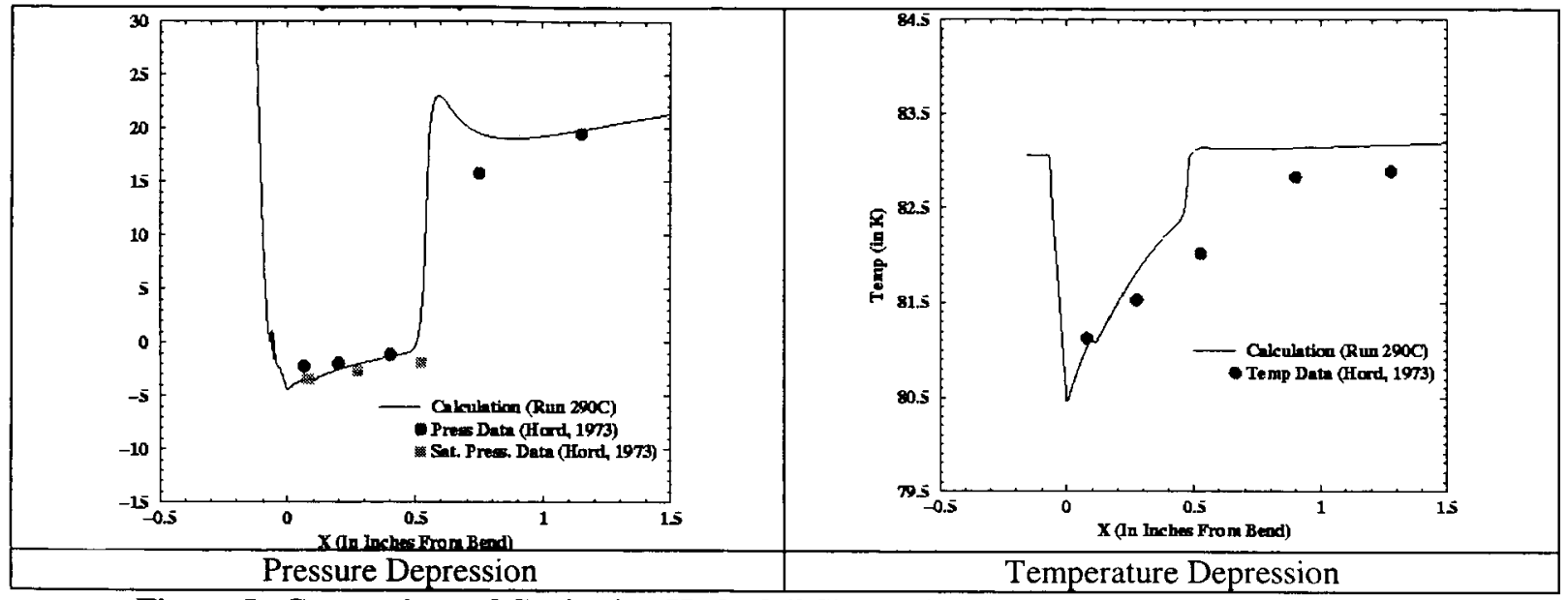

Figure 5. Comparison of Cavitating Hydrofoil Simulations with Data for Liquid Nitrogen.

The comparison of the pressure depression in Fig. 5a also indicates excellent overall comparison with data and within the instrumentation error bar of $0.69 \mathrm{~N} / \mathrm{cm}^{2}$. The leading edge pressure depression is 23.5 percent relative to the freestream vapor pressure. It further illustrates why temperature effects have a substantial impact on the performance of cryogenic pumps. The experimental data plotted includes both the actual pressure measured (symbol: circle) as well as the saturation pressure values (symbol: square) corresponding to the temperature measurements. The close match between the actual pressure and saturation pressure values indicates that the thermodynamic equilibrium assumption is valid for liquid nitrogen flows. .

\section{Inducer Simulations at Low-Flow Conditions}

The inducer chosen for our validation study is a three-bladed liquid hydrogen inducer designed at NASA Marshall (Thornton 16) using the Agile Engineering Design System tool developed by ConceptsNREC. The subscale configuration ( 0.37 scale) tested in water b ConceptsNREC had a diameter of 4.394 inches, a tip clearance of 0.014 inches, a shaft speed of $3840 \mathrm{rpm}$, and a flow rate of $195 \mathrm{gpm}$ at the design point. Figure 6 illustrates the inducer blade angle, inducer wrap and blade thickness distribution as a function of meridional length.

Figure 7 shows the three-dimensional geometry along with features of the multi-element unstructured grid that was used. In general, hexahedral cells are used around each blade while the region in between the hexahedral blocks is filled with tetrahedral cells. This strategy yields high-quality grids with minimal skewness even for extremely shallow blade angles that are typically associated with inducer blades.

We present simulation results for three flow rates; $235 \mathrm{gpm}$ (120\% of design), $195 \mathrm{gpm}$ (design), and $155 \mathrm{gpm}$ ( $80 \%$ of design). Note that the inducer test showed large scale separation even at the design condition of $195 \mathrm{gpm}$ while the $80 \%$ case obviously had a much larger separation zone. No backflow is observed at the $120 \%$ case of 235 gpm. Detailed radial profiles of both the meridional velocity and swirl velocity as well as the pressure were measured 2.44 inches upstream of the leading edge tip location. The upstream experimental data was used to estimate the velocity profiles at the leading edge of the blades by correcting for the hub area ad by preserving conservation of mass and angular momentum along stream tubes (Thornton ${ }^{16}$ ). Note that in the instances where we 
compare simulations with the leading edge "data" the simulation profiles are the actual computed solution, while the "data" is in fact the estimate from the raw data half a diameter upstream.

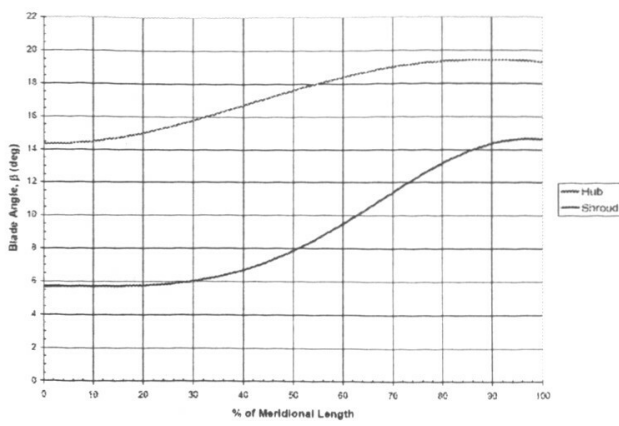

(a)

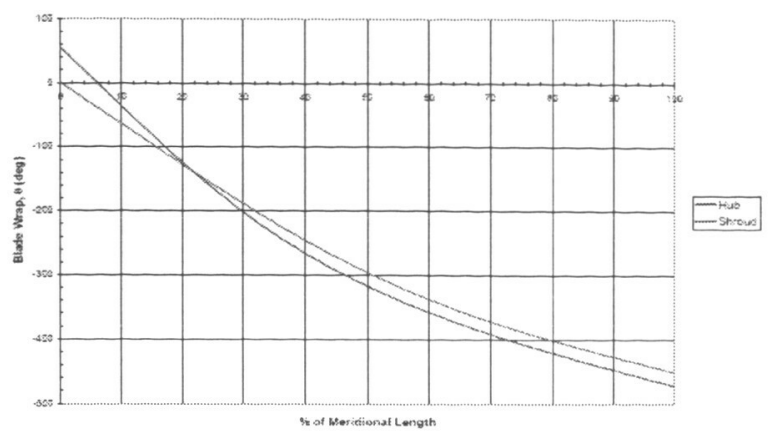

(b)

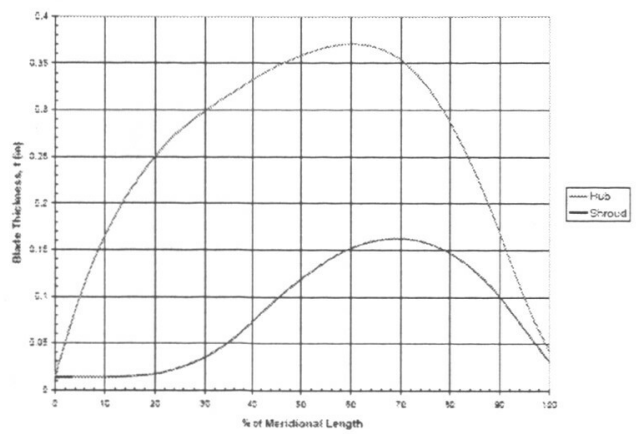

(c)

Figure 6. Inducer Blade Design Parameter Variation with Meridional Length: a) Blade angle; b) Blade Wrap; and c) Blade Thickness (Taken From Ref. 16).

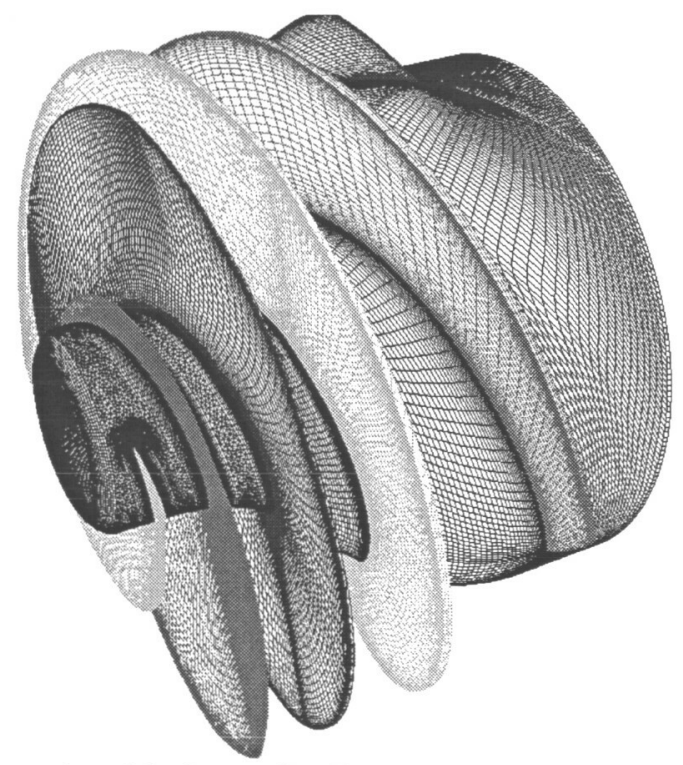

Figure 7. Three-Dimensional Inducer Configuration and Multi-Element grid Topology.

We begin by presenting results for the $235 \mathrm{gpm}(120 \%)$ case for which no back flow is observed. The velocity and pressure profiles upstream in the inlet are uniform and hence we compare the profiles for the meridional velocity and incidence angles at the leading edge with the approximate 1-D procedure in Figure 8. Good comparison is obtained for both quantities and as we shall see from comparisons with the upstream data for other 
flow conditions, the minor differences observed are more likely due to the 1-D approximations used to process the experimental data.

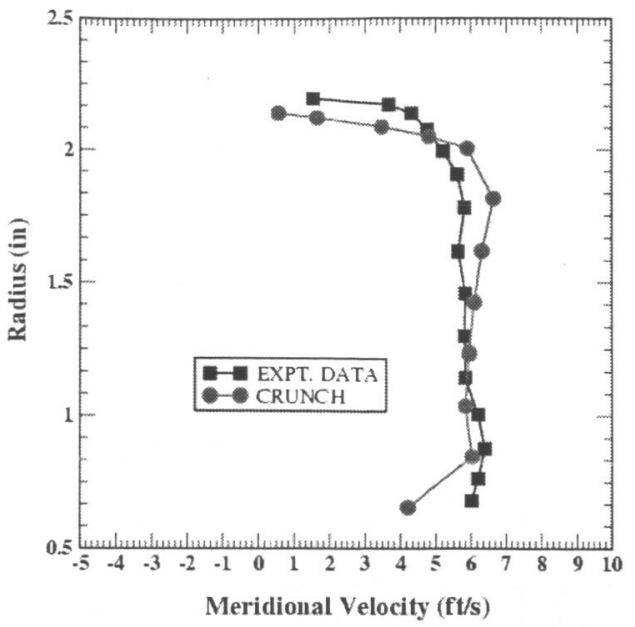

(a)

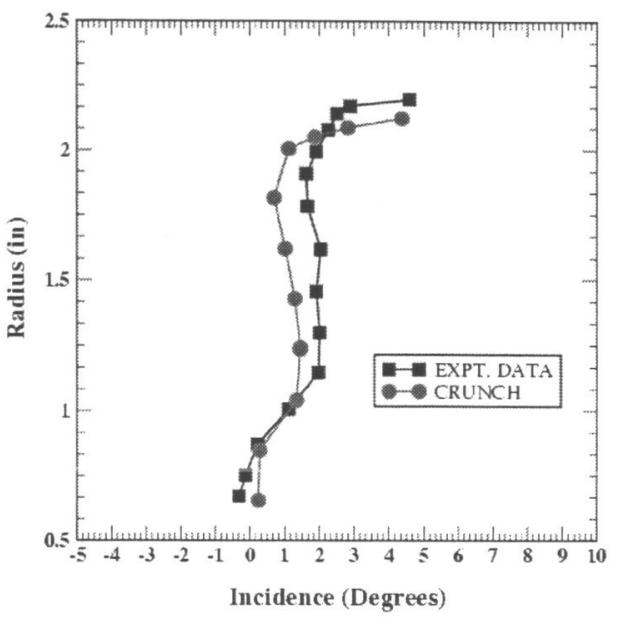

(b)

Figure 8. Blade LeadingEdge Velocity Profiles for $120 \%$ of Design Flow Rate:

a) Merdional Velocity; b) Incidence Angle.

The results for the design condition of $195 \mathrm{gpm}$ indicate that separation occurs at the leading edge with a substantial back flow region as is evident from the flow contours of the meridional velocity, pressure and the effective turbulent viscosity shown in Figure 9. A comparison of the radial flow profiles in Figure 10 with experimental data indicate that while the qualitative nature of the profiles are similar the extent of the back flow is underpredicted resulting in lower swirl velocities and higher values for pressure in the core relative to the shroud. The smaller extent of the back flow was found to result from an over prediction of the turbulent viscosity from the turbulence models that damped the reverse flow and not from numerical issues such as grid resolution and location of inflow boundary.

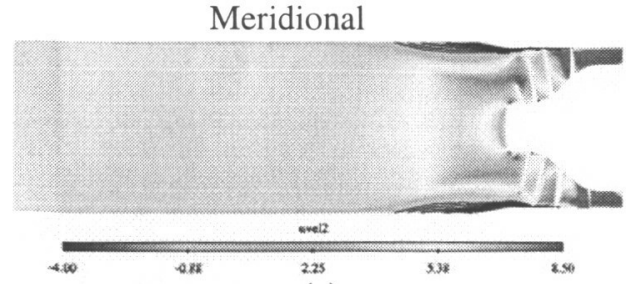

(a)

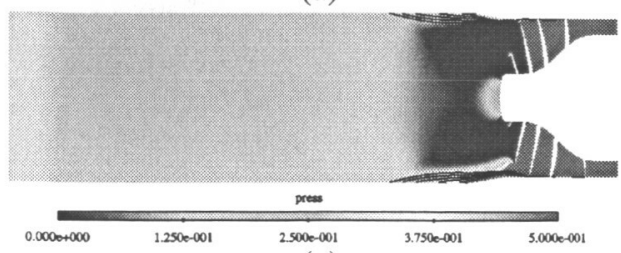

(c)

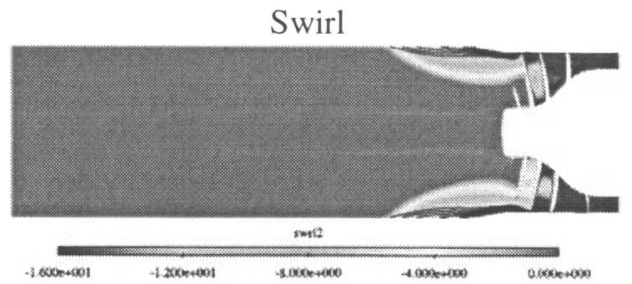

(b)

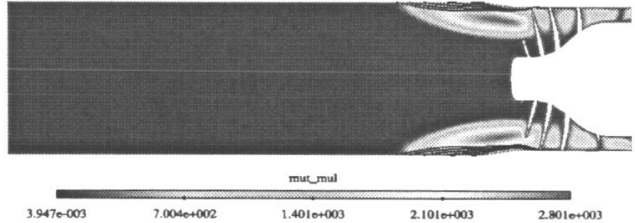

(d)

Figure 9. Flow Contours for $100 \%$ of Design Flow Rate; a) Meridional Velocity, b) Swirl Velocity, c) Pressure, d) Turbulent Viscosity.

A sensitivity study to the turbulent viscosity levels was undertaken by reducing the constant $c_{\mu}$. As the constant is reduced from 0.09 to 0.06 and then to 0.03 we observe (from Figure 10) that the meridional velocity and swirl velocity profiles compare well with the experimental data while the pressure comparison is reasonable. The flow contours for $c_{\mu}$ of 0.03 indicate a separation zone almost twice the original separation length and turbulent viscosity levels that are roughly half the baseline case (Figure 11). 
The meridional velocity and incidence angle profile at the leading edge are plotted in Figure 12 and they reveal an extremely interesting point: under non-cavitating conditions the velocity profiles and incidence angles at the blade leading edge are relatively insensitive to errors in the prediction of the separation zone. These results also indicate that the 1-D stream tube approximations to obtain the profile at the leading breakdown breakdown when separation occurs. The insensitivity of the leading edge profiles to the separation zone length would indicate that the single-phase performance predictions would not be affected significantly by errors in the estimation of the back flow but clearly cavitation performance would be quite sensitive to these errors since the extent of separation impacts the pressure depression in the core. Studies with other turbulence models that may perform better under swirling conditions (e.g. realizable k- $\varepsilon$ model) are currently underway.

Simulations at $155 \mathrm{gpm}$ (80\% of design) were carried out with the $c_{\mu}$ value at 0.03 which yielded the best results at the design flow rate. Figure 13 shows the corresponding flow contours for meridional velocity, swirl velocity, and pressure. The back flow zone is substantially larger and extends nearly 3 diameters downstream.

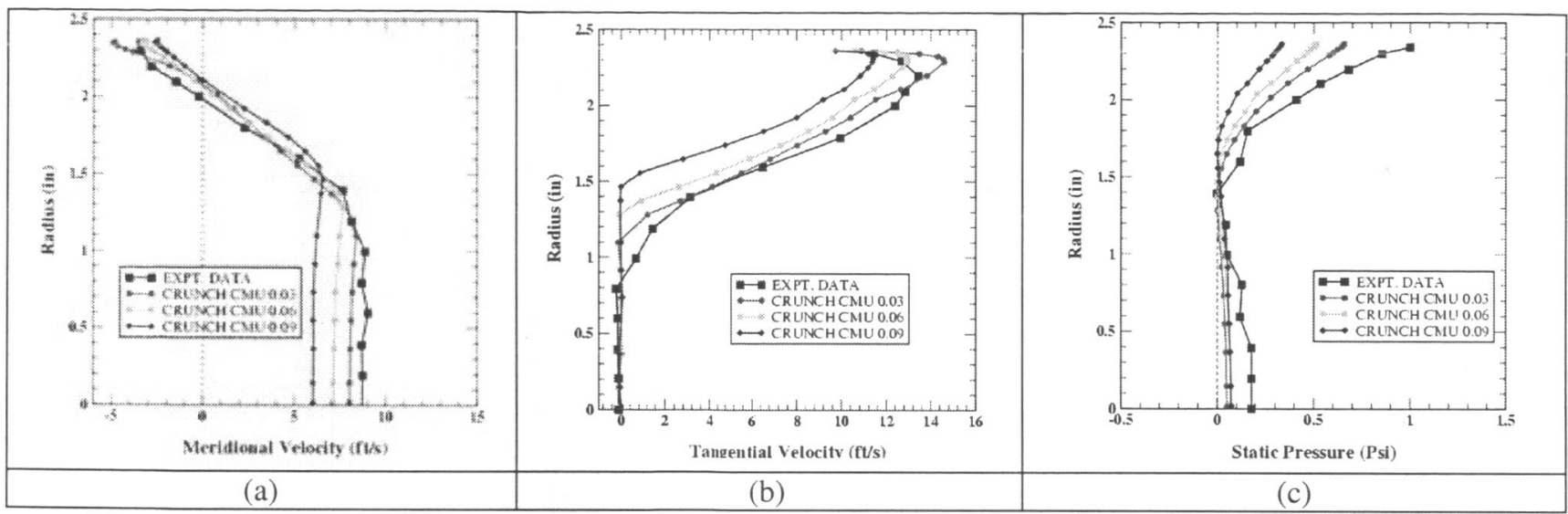

Figure 10. Radial Flow profiles compared with Data for $100 \%$ of Design flow Rate Half a Diameter Upstream of Leading Edge: a) Meridional Velocity; b) Swirl Velocity; and c) Pressure.

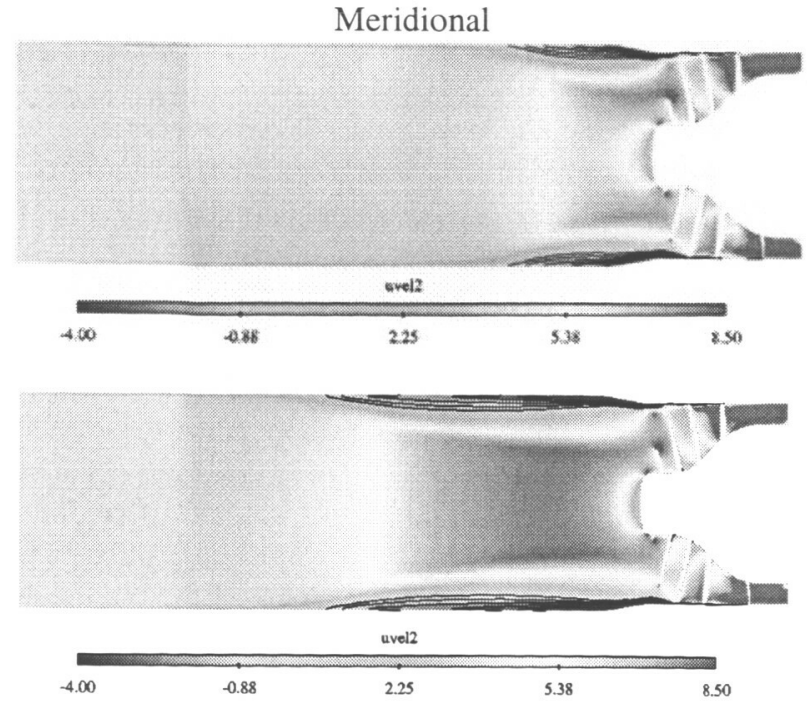

(a). $\mathrm{C} \mu=0.09$
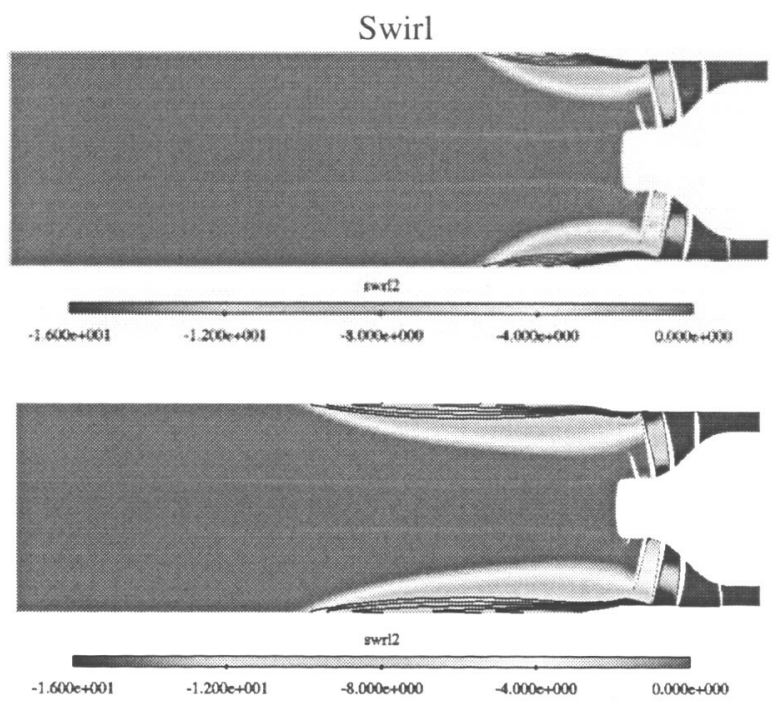

(b). $\mathrm{C} \mu=0.03$

Figure 11. Sensitivity of Flow Contours to Turbulent Viscosity a): $C \mu=0.09 ; b) C \mu=0.03$. 

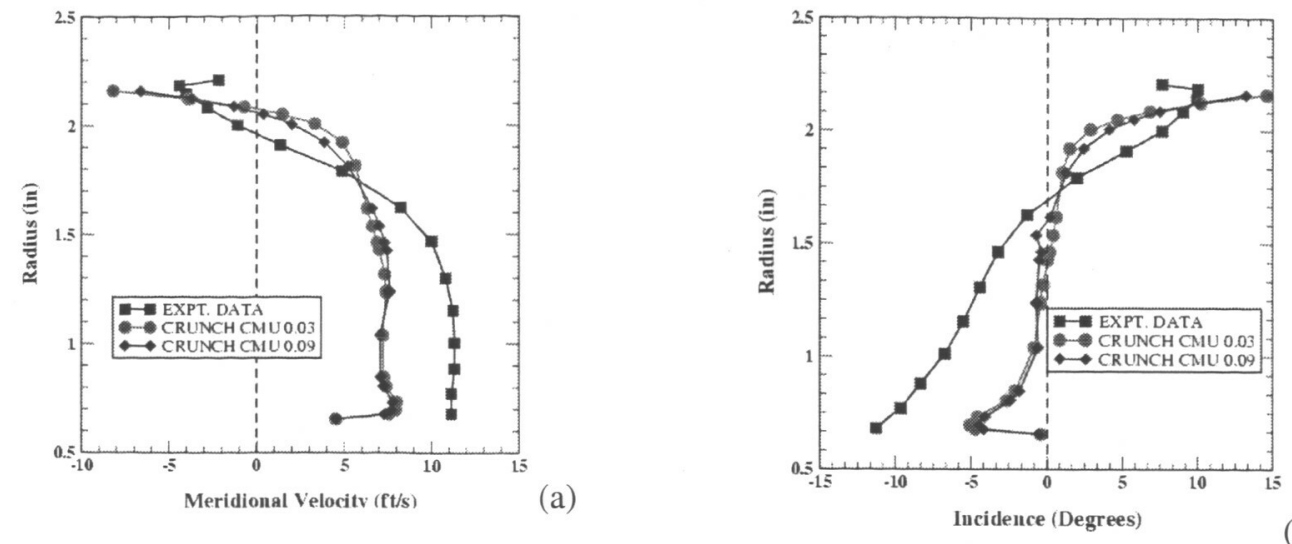

(b)

Figure 12. Blade Leading Edge profiles for $100 \%$ of Design Flow Rate: a) Meridional Velocity; b) Incidence Angle.

The radial profiles for the velocity and pressure are compared with experimental data in Figure 14. Excellent comparison is obtained for the pressure and swirl components in particular. The back flow has high swirl near the shroud of approximately $18 \mathrm{ft} / \mathrm{s}$ which in turn generates a pressure gradient of 2.5 psi between the shroud and the core. This low pressure core will clearly result in a cavitating core as the inlet pressure is dropped and possibly lead to cavitation induced axial instabilities. Finally we present the head coefficient computed for the three flow rates modeled with the experimental data in Figure 15. Good comparison is obtained for all three cases indicating that the inducer performance is being simulated well.

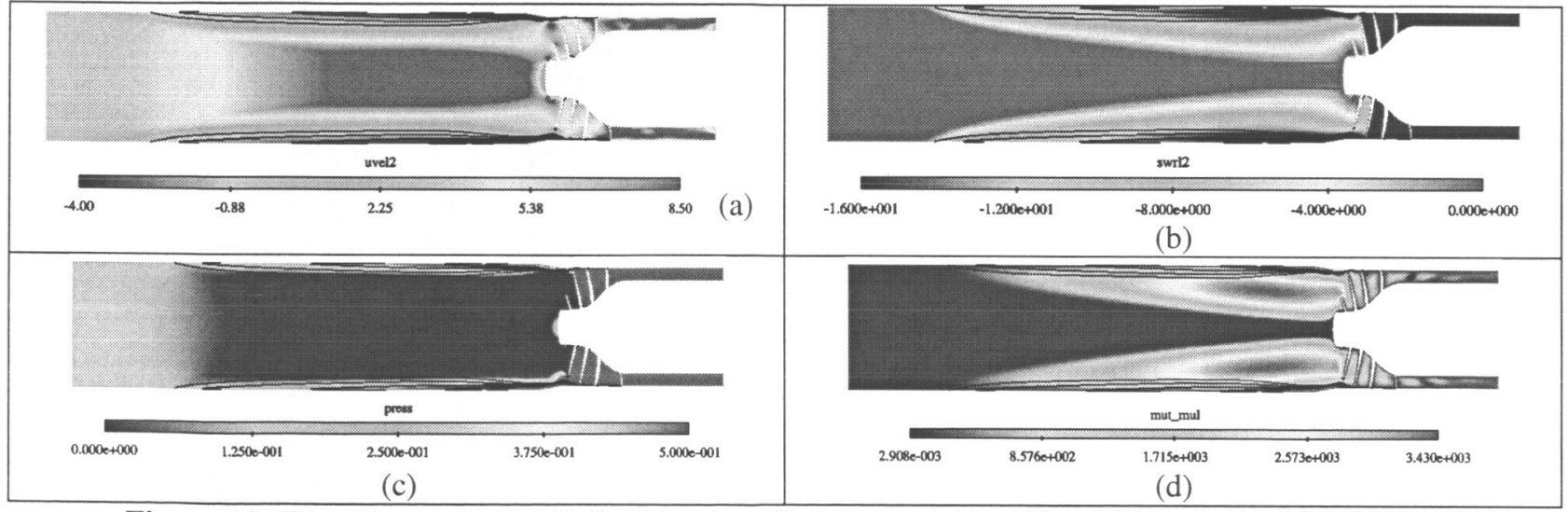

Figure 13. Flow Contours for $80 \%$ of Design Flow Rate: a) Meridional Velocity; b) Swirl Velocity; c) Pressure; d) Turbulent Viscosity.

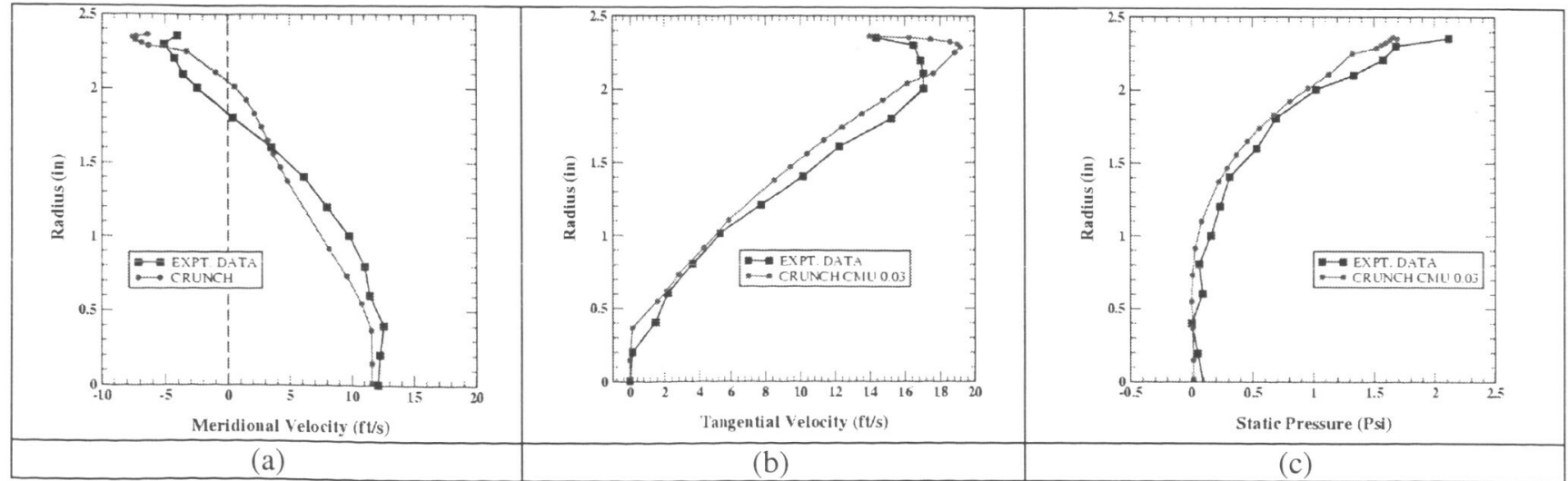

Figure 14. Radial Flow profiles compared with Data for $80 \%$ of Design flow Rate Half a Diameter Upstream of Leading Edge: a) Meridional Velocity; b) Swirl Velocity; and c) Pressure. 


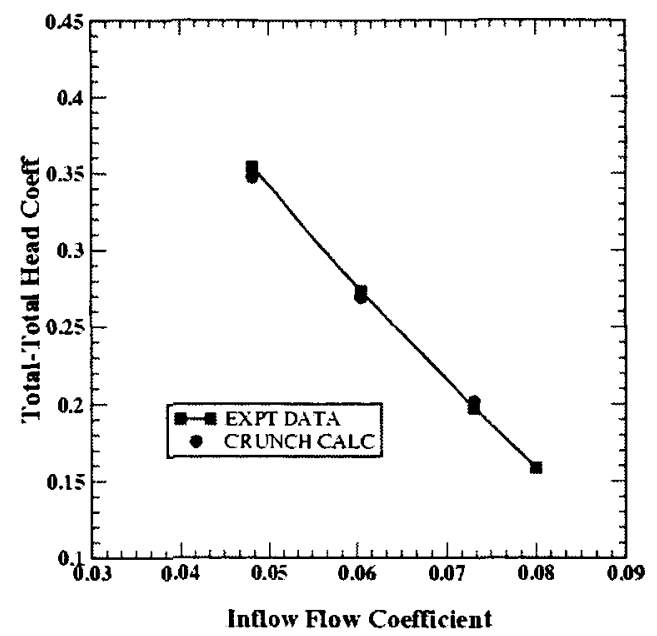

Figure 15. Computed Head Coefficient Compared with Experimental Data for Various Flow Coefficients.

\section{Conclusion}

A generalized multi-phase formulation has been developed for cavitation in "real" fluid flows (e.g., cryogenic fluids) that operate at temperatures close to their critical temperatures. It has also been previously shown to predict the cavitation breakdown point for inducers at design conditions where substantial back flow is not present.

The focus of the current effort is in continued validation of this tool for predicting off-design performance of inducers. A sub-scale version of a three-bladed liquid hydrogen inducer tested in water with detailed velocity and pressure measurements was used as a numbered test bed. Under low-flow, off-design conditions it was found that the length of the separation zone as well as the swirl velocity magnitude was under predicted with a standard $k-\varepsilon$ model and the turbulent viscosity constant had to be reduced. With the modified constant good comparison was obtained at all the flow conditions examined with both the magnitude and shape of the profile matching well with the experimental data taken half a diameter upstream of the leading edge. The velocity profiles and incidence angles at the leading edge itself were less sensitive to the back flow length predictions indicating that single-phase performance predictions may be well predicted even if the details of flow separation modeled are incorrect. However, for cavitating flow situations the prediction of the correct swirl in the back flow and the pressure depression in the core becomes critical since it leads to vapor formation in the core that can couple with the hydrodynamics and generate cavitation surge.

\section{Acknowledgments}

We acknowledge funding for this work through a SBIR program under Contract No.: NAS8-02098 funded by NASA Marshall Space Flight Center. The contract monitor is Dr. Dan Dorney. The technical inputs provided by Dr. Dan Dorney and Mr. Robert Garcia are gratefully acknowledged. The authors would also like to thank Dr Paul Cooper for his help and advice.

\section{References}

1 Hord, J., "Cavitation in Liquid Cryogens," NASA CR-2156, January 1973.

2 Stahl, H.A. and Stepanoff, A.J., "Thermodynamic Aspects of Cavitation in Centrifugal Pumps," ASME J. Basic Eng., Vol. 78, 1956, pp. 1691-1693.

3 Ruggeri, S.R., and Moore, R.D., "Method for Prediction of Pump Cavitation Performance for Various Liquids, Liquid Temperature, and Rotation Speeds, NASA TND-5292, 1969.

4 Holl, J.W., Billet M.L., and Weir, D.S., "Thermodynamic Effects On Developed Cavitation," ASME J. Fluids Eng., Vol. 97, No.4, pp. 507-516, 1975.

5 Brennen, C.E., "The Dynamic Behavior and Compliance of a Stream of Cavitating Bubbles," Journal of Fluids Engineering, pp. 533-542, Vol. 95, 1973.

6 Cooper, P., "Analysis of Single and Two-Phase Flows in Turbopump Inducers," Journal of Engineering for Power, Transactions of the ASME, 1967, pp. 577-588.

7 Hosangadi, A., Ahuja, V., and Ungewitter, R.J. "Simulations Of Cavitating Flows In Turbopumps," Journal of Propulsion and Power, Vol. 20, No. 4, pp. 604-611, July-August, 2004. 
Athavale, M.M. and Singhal, A.K., "Numerical Analysis of Cavitating Flows in Rocket Turbopump Elements," $37^{\text {th }}$ JPC, Salt Lake City, UT, July 2001.

9 Dupont, P., and Okamura, T., "Cavitating Flow Calculations in Industry", The $9^{\text {th }}$ International Symposium on Transport Phenomena and Dynamics of Rotating Turbomachinery, Honolulu, Hawaii, 2002.

10 Medvitz, R.B., Kunz, R.F., Boger, D.A., Lindau, J.W., Yocum, A.M., Pauley, L.L., "Performance Analysis of Cavitating Flow in Centrifugal Pumps Using Multi-Phase CFD”, Journal Fluids Engg., Vol. 124, 2002, pp. 377-383.

1 Hosangadi, A. and Ahuja, V., "A Numerical Study Of Cavitation In Cryogenic Fluids," Journal of Fluids Engg., To be Published March 2005.

12 Ahuja, V., Hosangadi, A. and Arunajatesan, S., "Simulations of Cavitating Flows Using Hybrid Unstructured Meshes," Journal of Fluids Engineering, May/June, 2001, Vol.123, pp 331-340.

13 Hosangadi, A., Lee, R.A., York, B.J., Sinha, N., and Dash, S.M., "Upwind Unstructured Scheme for Three-Dimensional Combusting Flows," Journal of Propulsion and Power, Vol. 12, No. 3, pp. 494-503, May-June 1996.

14 Hosangadi, A., Lee, R.A., Cavallo, P.A., Sinha, N., and York, B.J., "Hybrid, Viscous, Unstructured Mesh Solver for Propulsive Applications," AIAA-98-3153, AIAA 34 ${ }^{\text {th }}$ JPC, Cleveland, OH, July 13-15, 1998.

15 Hosangadi, A., Ahuja, V., and Ungewitter, R.J. "Simulations Of Cavitation Cryogenic Inducers," Paper No. AIAA-2004-4023, 40th AIAA/ASME /SAE/ASEE Joint Propulsion Conf. and Exhibit, Fort Lauderdale, FL, July 11-14, 2004.

16 Thornton, R.J., "Design of a Low Pressure Liquid Hydrogen Inducer With the Agile Engineering Design System," NASA Marshall Technical Report, TD 61, Turbomachinery Functional Design Group, March 2003.

17 Japikse, D. and Baun, D., "Inducer Design and Performance," MSFC Fall Fluids Workshop, November 19-21, 2002.

18 Ruggeri, R.S., "Experimental Studies on Thermodynamic Effects of Developed Cavitation," Proc. International Symposium on the Fluid Mechanics and Design of Turbomachinery, Penn State University, University park, PA, Aug. - Sept. 1970.

19 Merkle, C.L., Feng, J.Z. and Buelow, P.E.O., "Computational Modeling of the Dynamics of Sheet Cavitation," Proceedings of the $3^{\text {rd }}$ International Symposium on Cavitation, Grenoble, 1998.

20 Sarosdy L.R. and Acosta, A.J., "Note on Observations of Cavitation in Different Fluids," Paper No. 60-WA-83, ASME Winter Annual Meeting, New York, Nov. 27- Dec. 2, 1960. 